\title{
Implicitization of Rational Parametric Curves and Surfaces
}

\author{
Michael Kalkbrener \\ Research Institute for Symbolic Computation (RISC) \\ Johannes Kepler University Linz, Austria
}

\begin{abstract}
In this paper we use Gröbner bases for the implicitization of rational parametric curves and surfaces in 3D-space. We prove that the implicit form of a curve or surface given by the rational parametrization

$$
x_{1}:=\frac{p_{1}}{q_{1}} \quad x_{2}:=\frac{p_{2}}{q_{2}} \quad x_{3}:=\frac{p_{3}}{q_{3}},
$$

where the $p$ 's and $q$ 's are univariate polynomials in $y_{1}$ or bivariate polynomials in $y_{1}, y_{2}$ over a field $K$, can always be found by computing

$$
G B\left(\left\{q_{1} \cdot x_{1}-p_{1}, q_{2} \cdot x_{2}-p_{2}, q_{3} \cdot x_{3}-p_{3}\right\}\right) \cap K\left[x_{1}, x_{2}, x_{3}\right],
$$

where $G B$ is the Gröbner basis with respect to the lexical ordering with $x_{1} \prec x_{2} \prec$ $x_{3} \prec y_{1} \prec y_{2}$, if for every $i, j \in\{1,2,3\}$ with $i \neq j$ the polynomials $p_{i}, q_{i}, p_{j}, q_{j}$ have no common zeros. This result leads immediately to an implicitization algorithm for arbitrary rational parametric curves.

Furthermore, we present an algorithm for the implicitization of arbitrary rational parametric surfaces and prove its termination and correctness.
\end{abstract}

\section{Introduction}

The automatic conversion of parametrically defined varieties into their implicit form is of fundamental importance in geometric modeling. The reason for this is that implicit and parametric representations are appropriate for different classes of problems. For instance, it is universally recognized that the parametric representation is best suited for generating points along a variety, whereas the implicit representation is most convenient for determining whether a given point lies on a specific variety. It is also well-known that the problem of intersecting two varieties is greatly simplified if one variety can be expressed implicitly and the other parametrically. 
For some time the implicitization problem has been deemed unsolvable in the CAD literature ([4] or [11]). In 1984 the problem has been solved for rational parametric curves in $2 \mathrm{D}$ and rational parametric surfaces in 3D by using resultants (see [10]). Resolvents have been applied to find the implicit representation of rational parametric cubic curves in 3D ([5]). Recently, algorithms based on resultants have been developed for solving the implicitization problem for rational parametric surfaces ([3] and [9]). Arnon and Sederberg used Gröbner bases for the implicitization of polynomial parametric varieties of dimension $n-1$ in $n$-dimensional space ([1]). In 1987 Buchberger generalized their method to the case of polynomial parametric varieties of arbitrary dimension ([2]). Recently, we applied Gröbner bases to the most general problem, the implicitization of rational parametric varieties of arbitrary dimension in arbitrary dimensional space ([8]). Many of the implicitization methods are highlighted in [7].

In this paper we use Gröbner bases for the implicitization of rational parametric curves and surfaces in 3D-space. In contrast to the algorithms for the implicitization of $m$-dimensional varieties in $n$-dimensional space presented in [8] the algorithms in this paper work without introducing new variables. Therefore they solve the implicitization problem in 3D-space much faster than the general algorithms. (A comparision of the computing times of our implementations in Maple can be found in [8]).

In this paper we prove that the implicit form of a curve or surface given by the rational parametrization

$$
x_{1}:=\frac{p_{1}}{q_{1}} \quad x_{2}:=\frac{p_{2}}{q_{2}} \quad x_{3}:=\frac{p_{3}}{q_{3}},
$$

where the $p$ 's and $q$ 's are univariate polynomials in $y_{1}$ or bivariate polynomials in $y_{1}, y_{2}$ over a field $K$, can always be found by computing

$$
G B\left(\left\{q_{1} \cdot x_{1}-p_{1}, q_{2} \cdot x_{2}-p_{2}, q_{3} \cdot x_{3}-p_{3}\right\}\right) \cap K\left[x_{1}, x_{2}, x_{3}\right],
$$

where $G B$ is the Gröbner basis with respect to the lexical ordering with $x_{1} \prec x_{2} \prec x_{3} \prec$ $y_{1} \prec y_{2}$, if for every $i, j \in\{1,2,3\}$ with $i \neq j$ the polynomials $p_{i}, q_{i}, p_{j}, q_{j}$ have no common zeros. Since we can always assume that $p_{i}$ and $q_{i}$ are relatively prime $(i=1,2,3)$, the above condition is always satisfied, if the $p$ 's and $q$ 's are univariate. Therefore, the above result leads immediately to an implicitization algorithm for arbitrary rational parametric curves.

Furthermore, we present an algorithm for the implicitization of arbitrary rational parametric surfaces and prove its termination and correctness.

In section 2 we state the problems we are concerned with. In section 3 a few theorems are proved which are necessary for showing the correctness of the algorithms, which we present in section 4 . 


\section{Problems}

Throughout the paper let $K$ be a field and $\bar{K}$ the algebraic closure of $K$.

Let $J$ be an ideal and $g_{1}, \ldots, g_{m}$ polynomials in $K\left[x_{1}, \ldots, x_{n}\right] . V(J)$ denotes the variety of $J$, i.e. the set

$$
\left\{a \in \bar{K}^{n} \mid f(a)=0 \text { for every } f \in J\right\} .
$$

Instead of $V\left(\operatorname{Ideal}\left(\left\{g_{1}, \ldots, g_{m}\right\}\right)\right)$ we will often write $V\left(\left\{g_{1}, \ldots, g_{m}\right\}\right)$.

Let $L$ be a field with $K \subseteq L$. Then $\left(a_{1}, \ldots, a_{n}\right) \in L^{n}$ is a generic point of $J$ if for every $f \in K\left[x_{1}, \ldots, x_{n}\right]:$

$$
f \in J \quad \text { iff } \quad f\left(a_{1}, \ldots, a_{n}\right)=0 .
$$

It is well-know that an ideal is prime if and only if it has a generic point with coordinates in a universal domain (see for instance [12]).

In this paper we want to solve the following two problems:

\section{Implicitization Problem for Rational Parametric Curves:}

given: rational parametrization of a curve

$$
x_{1}=\frac{p_{1}}{q_{1}} \quad x_{2}=\frac{p_{2}}{q_{2}} \quad x_{3}=\frac{p_{3}}{q_{3}},
$$

where $p_{1}, p_{2}, p_{3} \in K\left[y_{1}\right], q_{1}, q_{2}, q_{3} \in K\left[y_{1}\right]-\{0\}$ and $p_{i}$ and $q_{i}$ are relatively prime $(i=$ $1,2,3)$.

find: implicit representation of this curve, i.e. polynomials $g_{1}, \ldots, g_{m}$ in $K\left[x_{1}, x_{2}, x_{3}\right]$ such that

$$
V\left(\left\{g_{1}, \ldots, g_{m}\right\}\right)=V\left(P^{\prime}\right)
$$

where $P^{\prime}$ is the prime ideal in $K\left[x_{1}, x_{2}, x_{3}\right]$ with

$$
\left(\frac{p_{1}}{q_{1}}, \frac{p_{2}}{q_{2}}, \frac{p_{3}}{q_{3}}\right) \in K\left(y_{1}\right)^{3}
$$

as generic point.

\section{Implicitization Problem for Rational Parametric Surfaces:}

given: rational parametrization

$$
x_{1}=\frac{p_{1}}{q_{1}} \quad x_{2}=\frac{p_{2}}{q_{2}} \quad x_{3}=\frac{p_{3}}{q_{3}},
$$

where $p_{1}, p_{2}, p_{3} \in K\left[y_{1}, y_{2}\right], q_{1}, q_{2}, q_{3} \in K\left[y_{1}, y_{2}\right]-\{0\}$ and $p_{i}$ and $q_{i}$ are relatively prime $(i=1,2,3)$.

decide: whether the parametric object is a surface, i.e. whether the transcendence degree of

$$
K\left(\frac{p_{1}}{q_{1}}, \frac{p_{2}}{q_{2}}, \frac{p_{3}}{q_{3}}\right)
$$


(over $K$ ) is 2 . In this case

find: implicit representation of this surface, i.e. a polynomial $g$ in $K\left[x_{1}, x_{2}, x_{3}\right]$ such that

$$
V(\{g\})=V\left(P^{\prime}\right)
$$

where $P^{\prime}$ is the prime ideal in $K\left[x_{1}, x_{2}, x_{3}\right]$ with

$$
\left(\frac{p_{1}}{q_{1}}, \frac{p_{2}}{q_{2}}, \frac{p_{3}}{q_{3}}\right) \in K\left(y_{1}, y_{2}\right)^{3}
$$

as generic point.

Example 1 For the rational parametrization

$$
x_{1}=\frac{2 y_{2}}{1+y_{1}^{2}+y_{2}^{2}} \quad x_{2}=\frac{2 y_{1} y_{2}}{1+y_{1}^{2}+y_{2}^{2}} \quad x_{3}=\frac{y_{2}^{2}-y_{1}^{2}-1}{1+y_{1}^{2}+y_{2}^{2}}
$$

the implicit representation

$$
x_{1}^{2}+x_{2}^{2}+x_{3}^{2}-1
$$

of the unit sphere is a solution of the above problem.

\section{Theorems}

Throughout the paper let $p_{1}, p_{2}, p_{3} \in K\left[y_{1}, y_{2}\right]$ and $q_{1}, q_{2}, q_{3} \in K\left[y_{1}, y_{2}\right]-\{0\}$ such that $p_{i}$ and $q_{i}$ are relatively prime $(i=1,2,3)$. Let

$$
\begin{gathered}
f_{1}:=q_{1} \cdot x_{1}-p_{1}, \quad f_{2}:=q_{2} \cdot x_{2}-p_{2}, \quad f_{3}:=q_{3} \cdot x_{3}-p_{3}, \\
I:=I d e a l\left(\left\{f_{1}, f_{2}, f_{3}\right\}\right) \text { in } K\left[x_{1}, x_{2}, x_{3}, y_{1}, y_{2}\right]
\end{gathered}
$$

and let $Q_{1}, \ldots, Q_{r}$ be primary ideals in $K\left[x_{1}, x_{2}, x_{3}, y_{1}, y_{2}\right]$ such that $Q_{1} \cap \ldots \cap Q_{r}$ is a reduced primary decomposition of $I$. Furthermore, $P$ denotes the prime ideal in the polynomial ring $K\left[x_{1}, x_{2}, x_{3}, y_{1}, y_{2}\right]$ which has

$$
\left(\frac{p_{1}}{q_{1}}, \frac{p_{2}}{q_{2}}, \frac{p_{3}}{q_{3}}, y_{1}, y_{2}\right) \in K\left(y_{1}, y_{2}\right)^{5}
$$

as generic point.

Theorem 1 There exists an $i \in\{1, \ldots, r\}$ with

$$
Q_{i}=P
$$

and for every $j \in\{1, \ldots, r\}-\{i\}$ :

$$
Q_{j} \cap K\left[y_{1}, y_{2}\right] \neq\{0\}
$$


Proof: In this proof we use the following notation:

For a given ideal $F$ in $K\left[x_{1}, x_{2}, x_{3}, y_{1}, y_{2}\right]$ the ideal in $K\left(y_{1}, y_{2}\right)\left[x_{1}, x_{2}, x_{3}\right]$ generated by $F$ is denoted by $F^{\star}$.

Obviously, $I^{\star}$ is a zero-dimensional prime ideal. By [6] p.92, there exists exactly one element $i$ of $\{1, \ldots, r\}$ with

$$
Q_{i} \cap K\left[y_{1}, y_{2}\right]=\{0\} .
$$

Furthermore, $I^{\star}=Q_{i}^{\star}$. By [6] p.47, $P^{\star}$ is a zero-dimensional prime ideal. As $I \subseteq P$,

$$
P^{\star}=I^{\star}=Q_{i}^{\star} .
$$

Using [6] p.92 again,

$$
Q_{i}=P
$$

For the rest of the paper let us assume that

$$
Q_{1}=P
$$

and that $Q_{2}, \ldots, Q_{r}$ are ordered in such a way that there exists a $v \in\{1, \ldots, r\}$ such that

$$
\begin{aligned}
& Q_{1}, \ldots, Q_{v} \text { are isolated primary components and } \\
& Q_{v+1}, \ldots, Q_{r} \text { are embedded primary components. }
\end{aligned}
$$

Obviously,

$$
V(I)=V\left(P_{1}\right) \cup \ldots \cup V\left(P_{v}\right),
$$

where $P_{i}$ is the radical of $Q_{i}$ for $i=1, \ldots, r$.

By Krull's Primidealkettensatz (see for instance [6] p.179),

$$
\operatorname{dim}\left(P_{j}\right) \geq 2 \quad(j=1, \ldots, v),
$$

where $\operatorname{dim}\left(P_{j}\right)$ denotes the dimension of $P_{j}$.

Definition: Let $\left(b_{1}, b_{2}\right) \in \bar{K}^{2}$. We denote the number of elements in the set

$$
\left\{i \in\{1,2,3\} \mid p_{i}\left(b_{1}, b_{2}\right)=q_{i}\left(b_{1}, b_{2}\right)=0\right\}
$$

by zero $\left(b_{1}, b_{2}\right)$.

Example 2 We consider again the parametrization

$$
x_{1}=\frac{2 y_{2}}{1+y_{1}^{2}+y_{2}^{2}} \quad x_{2}=\frac{2 y_{1} y_{2}}{1+y_{1}^{2}+y_{2}^{2}} \quad x_{3}=\frac{y_{2}^{2}-y_{1}^{2}-1}{1+y_{1}^{2}+y_{2}^{2}}
$$

of the unit sphere. Then for $(0,0),(i, 0) \in \bar{Q}^{2}$, where $Q$ denotes the field of rational numbers:

$$
z \operatorname{ero}(0,0)=0 \quad \text { and } \quad z e r o(i, 0)=3
$$


Theorem 2 Let $j \in\{2, \ldots, v\}$ and $\left(a_{1}, a_{2}, a_{3}, b_{1}, b_{2}\right)$ the generic point of the prime ideal $P_{j}$ in $K\left[x_{1}, x_{2}, x_{3}, y_{1}, y_{2}\right]$. Then

$$
b_{1}, b_{2} \in \bar{K} \text { and } \operatorname{dim}\left(P_{j}\right) \leq z \operatorname{ero}\left(b_{1}, b_{2}\right) \text {. }
$$

Proof: First of all, we know from Theorem 1 that the transcendence degree of $K\left(b_{1}, b_{2}\right)$ is smaller than 2 .

Let us assume that the transcendence degree of $K\left(b_{1}, b_{2}\right)$ is 1 .

Let $i \in\{1,2,3\}$. From the fact that $p_{i}, q_{i}$ are relatively prime it follows that $\left(b_{1}, b_{2}\right)$ is no common zero of $p_{i}$ and $q_{i}$. As $f_{i}$ is an element of $P_{j}, a_{i}$ is algebraically dependent on $\left\{b_{1}, b_{2}\right\}$. Thus, $\operatorname{dim}\left(P_{j}\right)=1$. This is a contradiction to $(2)$.

Therefore,

$$
b_{1}, b_{2} \in \bar{K}
$$

If $\left(b_{1}, b_{2}\right)$ is no common zero of $p_{i}$ and $q_{i}$ then $a_{i}$ is algebraically dependent on $\left\{b_{1}, b_{2}\right\}$. Thus, the transcendence degree of $K\left(a_{1}, a_{2}, a_{3}, b_{1}, b_{2}\right)$ is less equal zero $\left(b_{1}, b_{2}\right)$. Therefore,

$$
\operatorname{dim}\left(P_{j}\right) \leq z \operatorname{ero}\left(b_{1}, b_{2}\right)
$$

\section{Theorem 3}

$$
\begin{gathered}
V(I) \neq V(P) \\
\text { implies }
\end{gathered}
$$

that there exists a $\left(b_{1}, b_{2}\right) \in \bar{K}^{2}$ with zero $\left(b_{1}, b_{2}\right) \geq 2$.

Proof: If $V(I) \neq V(P)$ then we obtain from (1) that $v$ is greater equal 2 . Let $\left(a_{1}, a_{2}, a_{3}, b_{1}, b_{2}\right)$ be the generic point of $P_{2}$. By Theorem 2 and (2),

$$
\left(b_{1}, b_{2}\right) \in \bar{K}^{2} \text { and zero }\left(b_{1}, b_{2}\right) \geq 2 \text {. }
$$

\section{Algorithms}

If for every $(i, j) \in\{(1,2),(1,3),(2,3)\}$

$p_{i}, q_{i}, p_{j}, q_{j}$ have no common zeros

then, by Theorem 3,

$$
V\left(I \cap K\left[x_{1}, x_{2}, x_{3}\right]\right)=V\left(P \cap K\left[x_{1}, x_{2}, x_{3}\right]\right) .
$$


In this case it follows from the elimination property of Gröbner bases that we can obtain the implicit form of the curve or the surface given by

$$
x_{1}=\frac{p_{1}}{q_{1}} \quad x_{2}=\frac{p_{2}}{q_{2}} \quad x_{3}=\frac{p_{3}}{q_{3}}
$$

by computing

$$
\left\{g_{1}, \ldots, g_{m}\right\}:=G B\left(\left\{q_{1} \cdot x_{1}-p_{1}, q_{2} \cdot x_{2}-p_{2}, q_{3} \cdot x_{3}-p_{3}\right\}\right) \cap K\left[x_{1}, x_{2}, x_{3}\right],
$$

where $G B$ has to be computed using the lexical ordering determined by $x_{1} \prec x_{2} \prec x_{3} \prec$ $y_{1} \prec y_{2}$.

In particular, if a polynomial parametric surface or a rational parametric curve is given we obtain from Theorem 3:

\section{Corollary 1}

a) (Parametrization by polynomial functions:)

If $q_{1}=q_{2}=q_{3}=1$ then $V(I)=V(P)$.

\section{b) (Rational parametrization of curves:)}

If $p_{1}, p_{2}, p_{3}, q_{1}, q_{2}, q_{3} \in K\left[y_{1}\right]$ then $V(I)=V(P)$.

Hence, the simple algorithm described above solves the implicitization problem for rational parametric curves.

It is an easy consequence of Theorem 2 that

$$
I \cap K\left[x_{1}, x_{2}, x_{3}\right]=\{0\}
$$

iff

there exists a $\left(b_{1}, b_{2}\right) \in \bar{K}^{2}$ with zero $\left(b_{1}, b_{2}\right)=3$.

Therefore, if there exists such a $\left(b_{1}, b_{2}\right)$ then every technique from elimination theory must fail in finding an implicit representation.

Example 3 The implicit equation of the unit sphere cannot be found by computing

$$
G B\left(\left\{q_{1} \cdot x_{1}-p_{1}, q_{2} \cdot x_{2}-p_{2}, q_{3} \cdot x_{3}-p_{3}\right\}\right) \cap K\left[x_{1}, x_{2}, x_{3}\right],
$$

where the p's and q's are defined as in Example 1 or 2:

Since there exists $a\left(b_{1}, b_{2}\right) \in \bar{K}^{2}$ with zero $\left(b_{1}, b_{2}\right)=3$ (see Example 2),

$$
\operatorname{Ideal}\left(\left\{q_{1} \cdot x_{1}-p_{1}, q_{2} \cdot x_{2}-p_{2}, q_{3} \cdot x_{3}-p_{3}\right\}\right) \cap K\left[x_{1}, x_{2}, x_{3}\right]=\{0\}
$$

and therefore

$$
G B\left(\left\{q_{1} \cdot x_{1}-p_{1}, q_{2} \cdot x_{2}-p_{2}, q_{3} \cdot x_{3}-p_{3}\right\}\right) \cap K\left[x_{1}, x_{2}, x_{3}\right]=\emptyset .
$$


The same problem is addressed in [3] and [9]. In these papers parametrizations of that kind are called parametrizations with base points. Resultant techniques are used to compute implicit representations.

In this paper we use Gröbner bases for solving the implicitization problem for rational parametric surfaces.

Definition: Let $h, g$ be polynomials in $K\left[x_{1}, x_{2}, x_{3}, y_{1}\right]$ such that $g$ has no non-trivial factor in $K\left[y_{1}\right]$ and there exists a polynomial $p$ in $K\left[y_{1}\right]$ with $h=g \cdot p$. Then

$$
h_{/ y_{1}}:=g
$$

implicit_surface (in: $p_{1}, p_{2}, p_{3}, q_{1}, q_{2}, q_{3}$; out: $g$ )

input: $p_{1}, p_{2}, p_{3} \in K\left[y_{1}, y_{2}\right], q_{1}, q_{2}, q_{3} \in K\left[y_{1}, y_{2}\right]-\{0\}$ and

$$
p_{i} \text { and } q_{i} \text { are relatively prime } \quad(i=1,2,3) \text {. }
$$

output: $g \in K\left[x_{1}, x_{2}, x_{3}\right]$ such that if the transcendence degree of

$$
K\left(\frac{p_{1}}{q_{1}}, \frac{p_{2}}{q_{2}}, \frac{p_{3}}{q_{3}}\right)
$$

is 2 then

$$
g \notin K \text { and } V(\{g\})=V\left(P^{\prime}\right),
$$

where $P^{\prime}$ is the prime ideal in $K\left[x_{1}, x_{2}, x_{3}\right]$ with the generic point

$$
\left(\frac{p_{1}}{q_{1}}, \frac{p_{2}}{q_{2}}, \frac{p_{3}}{q_{3}}\right)
$$

and

$$
g=1
$$

otherwise.

$$
\begin{aligned}
& \text { for every }(i, j) \in\{(1,2),(1,3),(2,3)\} \text { do } \\
& \qquad \begin{array}{l}
G_{(i, j)}:=G B\left(\left\{f_{i}, f_{j}\right\}\right) \cap K\left[x_{1}, x_{2}, x_{3}, y_{1}\right], \text { where } f_{k}:=q_{k} \cdot x_{k}-p_{k} \quad(k=1,2,3) \\
\quad F_{(i, j)}:=\left\{h_{/ y_{1}} \mid h \in G_{(i, j)}\right\} \\
G:=G B\left(F_{(1,2)} \cup F_{(1,3)} \cup F_{(2,3)} \cup\left\{f_{1}, f_{2}, f_{3}\right\}\right) \cap K\left[x_{1}, x_{2}, x_{3}\right] \\
g:=\operatorname{gcd}(G)
\end{array}
\end{aligned}
$$

where $G B$ has to be computed using the lexical ordering determined by $x_{1} \prec x_{2} \prec x_{3} \prec$ $y_{1} \prec y_{2}$. 
Example 4 Again we consider the unit sphere given by

$$
x_{1}=\frac{2 y_{2}}{1+y_{1}^{2}+y_{2}^{2}} \quad x_{2}=\frac{2 y_{1} y_{2}}{1+y_{1}^{2}+y_{2}^{2}} \quad x_{3}=\frac{y_{2}^{2}-y_{1}^{2}-1}{1+y_{1}^{2}+y_{2}^{2}} .
$$

Using implicit_surface we obtain

$G_{(1,2)}:=\left\{x_{2}+y_{1}^{2} x_{2}-x_{1} y_{1}-y_{1}^{3} x_{1}\right\}$,

$F_{(1,2)}:=\left\{-x_{2}+x_{1} y_{1}\right\}$,

$G_{(1,3)}:=\left\{x_{1}^{2}+2 x_{1}^{2} y_{1}^{2}-y_{1}^{2}-1+y_{1}^{4} x_{1}^{2}+x_{3}^{2}+y_{1}^{2} x_{3}^{2}\right\}$,

$F_{(1,3)}:=\left\{x_{1}^{2} y_{1}^{2}+x_{1}^{2}-1+x_{3}^{2}\right\}$,

$G_{(2,3)}:=\left\{-x_{2}^{2}-2 y_{1}^{2} x_{2}^{2}+y_{1}^{4}+y_{1}^{2}-y_{1}^{4} x_{2}^{2}-y_{1}^{2} x_{3}^{2}-y_{1}^{4} x_{3}^{2}\right\}$,

$F_{(2,3)}:=\left\{y_{1}^{2} x_{2}^{2}+x_{2}^{2}-y_{1}^{2}+y_{1}^{2} x_{3}^{2}\right\}$,

$G:=\left\{x_{1}^{2}+x_{2}^{2}+x_{3}^{2}-1\right\}$,

$g:=x_{1}^{2}+x_{2}^{2}+x_{3}^{2}-1$, the implicit representation of the unit sphere.

As termination of the algorithm is obvious it remains to prove its correctness.

\section{Proof of correctness:}

Let

$$
\bar{I}:=\operatorname{Ideal}\left(F_{(1,2)} \cup F_{(1,3)} \cup F_{(2,3)} \cup\left\{f_{1}, f_{2}, f_{3}\right\}\right),
$$

$\bar{P}$ a prime ideal in $K\left[x_{1}, x_{2}, x_{3}, y_{1}, y_{2}\right]$ with $\bar{I} \subseteq \bar{P}$ and $P \neq \bar{P}$ and let $\left(a_{1}, a_{2}, a_{3}, b_{1}, b_{2}\right)$ be the generic point of $\bar{P}$.

Assumption: $\operatorname{dim}(\bar{P})>1$.

Then,

$$
P \nsubseteq \bar{P}
$$

As $I \subseteq \bar{I} \subseteq \bar{P}$ there exists an $i \in\{2, \ldots, v\}$ with $P_{i} \subseteq \bar{P}$. By Theorem 2,

$$
b_{1}, b_{2} \in \bar{K}
$$

As $\operatorname{dim}(\bar{P})>1$ there exist $j, k \in\{1,2,3\}$ such that $j \neq k$ and $\left\{a_{j}, a_{k}\right\}$ is algebraically independent over $K$. Since $p_{j}$ and $q_{j}$ are relatively prime and $\operatorname{gcd}\left(f_{j}, f_{k}\right)$ divides $p_{j}$ and $q_{j}$,

$$
\operatorname{gcd}\left(f_{j}, f_{k}\right)=1
$$

Thus, Ideal $\left(\left\{f_{j}, f_{k}\right\}\right) \cap K\left[x_{1}, x_{2}, x_{3}, y_{1}\right] \neq\{0\}$ and therefore there exists a non-zero polynomial $f\left(x_{j}, x_{k}, y_{1}\right) \in F_{(j, k)}$. By definition of $F_{(j, k)}$,

$$
f\left(x_{j}, x_{k}, b_{1}\right) \neq 0 \text {. }
$$

This is a contradiction to the fact that $\left\{a_{j}, a_{k}\right\}$ is algebraically independent over $K$. 
Thus, $P$ is the only prime ideal that is a superideal of $\bar{I}$ and has a dimension greater than 1. Hence, $\bar{I}$ can be written in the form

$$
P \cap R
$$

where $R$ is an ideal in $K\left[x_{1}, x_{2}, x_{3}, y_{1}, y_{2}\right]$ with $\operatorname{dim}(R)<2$. Therefore,

$\bar{I} \cap K\left[x_{1}, x_{2}, x_{3}\right]=\left(P \cap K\left[x_{1}, x_{2}, x_{3}\right]\right) \cap\left(R \cap K\left[x_{1}, x_{2}, x_{3}\right]\right)$ and $\operatorname{dim}\left(R \cap K\left[x_{1}, x_{2}, x_{3}\right]\right)<2$.

It follows from the elimination property of Gröbner bases that

$$
G \text { is a basis of } \bar{I} \cap K\left[x_{1}, x_{2}, x_{3}\right] \text {. }
$$

Case:

$$
\text { the transcendence degree of } K\left(\frac{p_{1}}{q_{1}}, \frac{p_{2}}{q_{2}}, \frac{p_{3}}{q_{3}}\right) \text { is } 2 \text {. }
$$

In this case $P \cap K\left[x_{1}, x_{2}, x_{3}\right]$ is a prime ideal of dimension 2. Thus, there exists an $h \in$ $K\left[x_{1}, x_{2}, x_{3}\right]-K$ with $\operatorname{Ideal}(\{h\})=P \cap K\left[x_{1}, x_{2}, x_{3}\right]$. As $G \subseteq P \cap K\left[x_{1}, x_{2}, x_{3}\right]$,

$$
h \text { divides } \operatorname{gcd}(G) \text {. }
$$

Let $p \in K\left[x_{1}, x_{2}, x_{3}\right]$ such that $\operatorname{gcd}(G)=h \cdot p$. Obviously, $p$ divides every polynomial in $R \cap K\left[x_{1}, x_{2}, x_{3}\right]$. As the dimension of $R \cap K\left[x_{1}, x_{2}, x_{3}\right]$ is less than $2, p$ is a non-zero constant. Thus,

$$
V(\{\operatorname{gcd}(G)\})=V(\{h\})=V\left(P \cap K\left[x_{1}, x_{2}, x_{3}\right]\right)=V\left(P^{\prime}\right) .
$$

Case:

$$
\text { the transcendence degree of } K\left(\frac{p_{1}}{q_{1}}, \frac{p_{2}}{q_{2}}, \frac{p_{3}}{q_{3}}\right) \text { is less than } 2 .
$$

In this case $\operatorname{dim}\left(P \cap K\left[x_{1}, x_{2}, x_{3}\right]\right)$ is less than 2 and therefore, by $(3), \operatorname{dim}\left(\bar{I} \cap K\left[x_{1}, x_{2}, x_{3}\right]\right)$ is less than 2. Hence,

$$
\operatorname{gcd}(G)=1
$$

Some of the Gröbner bases computations in implicit_surface can be replaced by other elimination methods, for instance by computations of Sylvester resultants:

We can replace

$$
G_{(i, j)}:=G B\left(\left\{f_{i}, f_{j}\right\}\right) \cap K\left[x_{1}, x_{2}, x_{3}, y_{1}\right]
$$

by

$G_{(i, j)}:=\left\{\operatorname{resultant}\left(f_{i}, f_{j}\right)\right\}$, where $f_{i}$ and $f_{j}$ are considered as polynomials in $y_{2}$.

Since resultants seem to have a better run-time behaviour, this could lead to a speed-up of the algorithm. 


\section{References}

[1] D.S. Arnon and T.W. Sederberg, Implicit Equation for a Parametric Surface by Gröbner Basis, in: V.E. Golden, ed., Proceedings of the 1984 MACSYMA User's Conference, General Electric, Schenectady, New York (1984) 431-436.

[2] B. Buchberger, Applications of Gröbner Bases in Non-Linear Computational Geometry, in: Proc. Workshop on Scientific Software, IMA, Minneapolis (1987).

[3] E.W. Chionh, Base Points, resultants, and the implicit representation of rational surfaces, Ph.D. thesis, Department of Computer Science, University of Waterloo, Canada (1990).

[4] I.D. Faux and M.A. Pratt, Computational Geometry for Design and Manufacture (Ellis Horwood, Chichester, 1981).

[5] R.N. Goldman, The method of resolvents: A technique for the implicitization, inversion, and intersection of non-planar, parametric, rational cubic curves, Computer Aided Geometric Design 2 (1985) 237-255.

[6] W. Gröbner, Algebraic Geometry II (Bibliographisches Institut Mannheim, 1970).

[7] C.M. Hoffmann, Geometric and Solid Modeling: An Introduction (Morgan Kaufmann Publishers Inc., 1989).

[8] M. Kalkbrener, Implicitization by Using Gröbner Bases, Technical Report RISC-Series 90-27, Univ. of Linz, Austria (1990).

[9] D. Manocha and J.F. Canny, Implicitizing Rational Parametric Surfaces, Technical Report UCB/CSD 90/592, Computer Science Division, University of California, Berkeley, California, U.S.A. (1990).

[10] T.W. Sederberg, D.C. Anderson, and R.N. Goldman, Implicit Representation of Parametric Curves and Surfaces, Computer Vision, Graphics, and Image Processing 28 (1984) $72-84$.

[11] H.G. Timmer, A Solution to the Surface Intersection Problem, Technical Report NAS2-9590, McDonnell-Douglas Corp. (1977).

[12] B.L. van der Waerden, Algebra II (Springer, Berlin Heidelberg New York, 1967). 